\title{
Cardiovascular Implantable Electronic Device Infections in Left Ventricular Assist Device Recipients
}

\author{
Talha Riaz, MD ${ }^{1}$, Juhsien JC Nienaber, MD ${ }^{1}$, Larry M. Baddour, MD¹, Randall C. Walker, \\ MD ${ }^{1}$, Soon J. Park, MD ${ }^{2}$, and M. Rizwan Sohail, MD ${ }^{1,3}$ \\ ${ }^{1}$ Division of Infectious Diseases, Mayo Clinic, Rochester, MN \\ ${ }^{2}$ Division of Cardiovascular Surgery, Mayo Clinic, Rochester, MN \\ ${ }^{3}$ Division of Cardiovascular Diseases, Mayo Clinic, Rochester, MN
}

\begin{abstract}
Background-Most patients with left ventricular assist devices (LVAD) have concomitant cardiovascular implantable electronic devices (CIED). However, clinical presentation and outcome of CIED infection in the setting of LVAD has not been previously described.
\end{abstract}

\begin{abstract}
Methods-We retrospectively reviewed 247 patients who underwent LVAD implantation at Mayo Clinic campuses in Minnesota, Arizona and Florida, from January 2005 to December 2011. Demographic and clinical data of patients that met criteria for CIED infection were extracted.

Results-Of 247 patients with LVADs, 215 (87\%) had CIED at the time of LVAD implantation and six (2.8\%) subsequently developed CIED infections. Three patients developed CIED leadrelated endocarditis and other 3 had pocket infection. All 3 instances of CIED pocket infection were preceded by device generator exchange, while all three patients with CIED lead-related endocarditis had prior LVAD-related infections. Causative pathogens included Pseudomonas aeruginosa (1), coagulase-negative staphylococci (2), methicillin-resistant Staphylococcus aureus (1), a gram-positive bacillus (1), and culture-negative (2). All patients underwent complete CIED removal along with antimicrobial therapy. The 3 patients with CIED lead- related endocarditis and prior LVAD infections received chronic suppressive antibiotic therapy, and one patient had LVAD exchange. All but one remained alive at the last follow-up with a median duration of 15 months (7-46 months) from the time of CIED infection.
\end{abstract}

Conclusion-Patients who are receiving LVAD therapy and develop CIED infection should be managed with complete CIED removal. Chronic suppressive antibiotic therapy is warranted in cases that have concomitant LVAD infection.

\section{Keywords}

Congestive Heart Failure; Defibrillation - ICD; CRT; Heart Transplantation

\section{INTRODUCTION}

With an aging population and advancements in the medical field, an increasing number of patients now undergo implantation of various cardiovascular devices, including prosthetic

Corresponding author: M. Rizwan Sohail MD, Division of Infectious Diseases, Mayo Clinic College of Medicine, 200 First Street SW, Rochester, MN 55905, 507-255-7767 (fax), sohail.muhammad@mayo.edu (primary), sohailmd@mac.com (alternative).

Conflicts of interest: None for all authors.

A poster abstract with preliminary results was presented at ACC, March 2013 
heart valves, cardiovascular implantable electronic devices (CIEDs), prosthetic vascular grafts and ventricular assist devices. Due to overlapping indications for some devices, patients may have more than one cardiovascular device. Of particular interest are those patients who have both a left ventricular assist device (LVAD) and a CIED, because advanced heart failure is a common indication for both devices [1] [2]. LVAD therapy is being increasingly used in end-stage heart failure patients, either as a bridge to transplant or as destination therapy. Since 2005, more than 7000 HeartMate II devices, the most widely used second generation LVAD model, have been implanted worldwide[2, 3]. Many patients receiving LVAD therapy also have CIEDs, which are often in place before the LVADs were implanted. In addition to rhythm management, combined electrical and mechanical support has been found beneficial in management of advanced heart failure[4].

A common complication shared by both of these foreign devices is the risk of infection. CIED infections are generally classified into local pocket infections and CIED- related endocarditis (CIED-IE) [5]. LVAD infections are similarly classified as local and endovascular infections. Local infections involve LVAD driveline and CIED pocket infections, while endovascular infections may present as sepsis, pump and cannula infection, bloodstream infection (BSI) or CIED lead-related, or LVAD-related endocarditis[6].

Patients with both LVAD and CIED may develop infection of one or both devices. Currently, there are no published data regarding clinical presentation and management of infected CIEDs in patients receiving concomitant LVAD therapy. In this report, we describe a case series of six patients who were receiving both LVAD and CIED therapy and presented with CIED infection. We discuss the clinical manifestations, management and outcome of these rather complex cases.

\section{METHODS}

We retrospectively reviewed 247 patients who underwent continuous-flow LVAD implantation (HeartMate II and Jarvik 2000) at Mayo Clinic campuses in Minnesota, Arizona, and Florida between January 2005 and December 2011. Patients in whom LVADs were not placed at Mayo Clinic, but subsequently followed at Mayo Clinic, and those with concurrent right ventricular assist device were excluded.

LVAD-related infections were classified based on recent consensus guidelines proposed by the International Society for Heart and Lung Transplantation[6]. CIED infection cases were classified as pocket infection or CIED-related endocarditis (CIED-IE) as previously described $[5,7,8]$. CIED-IE was defined by persistent bloodstream infections (BSI) and CIED lead vegetation on echocardiography [5, 9]. BSI was defined using Center for Disease Control's National Healthcare Safety Network (CDC/NHSN) definitions [10].

\section{ILLUSTRATIVE CASE}

A 72 year-old man (case 1) with a history of diabetes mellitus, chronic kidney disease, cerebrovascular disease (on chronic anticoagulation), and ischemic cardiomyopathy, had a dual-chamber implantable cardioverter-defibrillator (ICD) placed in 2003 and underwent LVAD implantation in 2010. Six months after LVAD implantation, he developed LVAD driveline infection and bacteremia with Pseudomonas aeruginosa that was treated with a 6week course of intravenous cefepime. He later required intraoperative debridement and marsupialization of the non-healing driveline exit site. However, he subsequently developed chronic driveline infection with multiple recurrences.

Twelve months after the initial episode of driveline infection and bacteremia with Pseudomonas, the patient had another episode of $P$. aeruginosa BSI that was treated with 
intravenous ceftazidime and gentamicin. A transesophageal echocardiography (TEE) was not performed at this time. However, towards the end of a planned 8-week course of systemic antibiotics, blood cultures turned positive again for multi-drug resistant $P$. aeruginosa and a TEE revealed an $18 \mathrm{~mm}$ vegetation on the atrial lead of his ICD. Due to multiple comorbidities, he was not deemed a surgical candidate for LVAD exchange and was medically managed with chronic suppression with intravenous meropenem, because the $P$. aeruginosa strain had become fluoroquinolone-resistant. While on the fourth week of intravenous meropenem therapy, he had a breakthrough BSI with this $P$. aeruginosa. An Indium-labeled white blood cells scan revealed infection involving the driveline, the ICD system, and the LVAD. Due to failure of conservative management, it was decided to remove the entire ICD system and debride the LVAD driveline and pump pocket. A temporary pacemaker was placed until the blood cultures (with the patient on antimicrobial therapy) became negative, and a new single-chamber pacemaker was implanted 7 days after the initial surgery. Although the initial plan was to perform LVAD exchange after an additional week of parenteral antibiotics after the new pacemaker, the treating medical team ultimately concluded that LVAD exchange would be too hazardous and unlikely to eradicate or successfully control the infection. Therefore, the patient was placed on indefinite intravenous ceftazidime and tobramycin suppression. Two months later, the patient presented with multiple cerebral hemorrhages, presumably due to septic emboli and was again bacteremic with $P$. aeruginosa. After discussion regarding goals of care and the poor prognosis, the patient and family opted for comfort care. The LVAD was turned off and the patient died later that day.

\section{RESULTS}

Between January 2005 and December 2011, 247 patients underwent continuous-flow LVAD implantation at Mayo Clinic. All patients received HeartMate II devices. Driveline exit sites for all were on the right. No patient had active infection at time of LVAD implantation, and none of the patients had any significant intraoperative complications, postoperative bleeding, or re-operation. All patients were anticoagulated per LVAD protocol. The majority of LVAD recipients $(215,87 \%)$ had an existing CIED at the time of LVAD implantation. Six of these patients developed CIED infections during median follow-up duration of one year. The key features of each case are summarized in Table 1 for comparison. Notably, overall follow-up duration was comparable between cases with CIED infection and those without it.

Median age of patients with CIED infection was 65 years (range, 35 to 72 years). There was only one female patient (of note, in the entire cohort of LVAD recipients, only 19\% [47/247] were females). All patients with CIED infection underwent LVAD implantation as destination therapy except one (case 3), where the LVAD was used as a bridge to transplantation.

Median time from LVAD implantation to CIED infection was 18 months (ranging from 3 to 32 months). Interestingly, all 3 patients who developed pocket infections had generator exchange procedure preceding their CIED infections. The median number of days from generator exchange to device infection was 18 days. Case 6 had the generator exchange elsewhere and this procedure was complicated by hematoma and subsequent wound dehiscence leading to pocket infection.

All cases with pocket infections had local erythema, tenderness, and warmth at the CIED generator pocket site. Four of the 6 patients had fever, including 3 cases with CIED-IE. Interestingly, peripheral white blood cell count was in the normal range in all patients except 
in case 3 where it was $14 \times 10^{9} / \mathrm{L}$. CIED-IE was diagnosed by TEE in 3 patients. Clinical presentation and microbiology of all cases are summarized in Table 1.

All 3 patients with CIED-IE had either prior or concurrent LVAD-related infection. Case 1 had chronic $P$. aeruginosa LVAD driveline infection. Case 2 had prior catheter-related BSI due to coagulase-negative staphylococcus (CoNS) and two episodes of LVAD driveline infections, with superficial driveline swab growing CoNS. Case 3 initially presented with BSI with methicillin-resistant $S$. aureus (MRSA) one month after LVAD implantation. He received 6 weeks of vancomycin and rifampin plus 2 weeks of gentamicin for presumed endocardial involvement of the LVAD (with TEE negative for endocarditis). However, this patient developed a relapse of MRSA BSI nearly 3 months post-LVAD implantation with vegetations noted on the CIED leads and prosthetic tricuspid valve. She had recurrent MRSA BSI due to persistent infections involving the LVAD, the pump pocket, and the driveline by what was later found to be small colony variant of MRSA. This patient received multiple courses of combination antibiotics therapy (including vancomycin, quinupristin/ dalfopristin, linezolid, gentamicin, rifampin, trimethoprim/sulfamethoxazole, and doxycycline in different combinations), but eventually underwent complete LVAD exchange (16 months after initial LVAD implantation) due to persistent infection. PostLVAD exchange surgery, the patient was then kept on chronic antibiotic suppression with trimethoprim/sulfamethoxazole and minocycline and was not yet transplanted at the last available follow-up due to antibody hypersensitization. As for those presenting with CIED pocket infections, device infection in case 4 was preceded by a polymicrobial catheterrelated BSI 9 months prior, while case 6 was followed by a possible driveline infection 10 month later.

Antimicrobial regimens used for CIED infection treatment are also summarized in table 1. All patients with CIED-IE received prolonged courses of antibiotics (minimum of 6 weeks) followed by chronic antibiotic suppression, whereas those with pocket infections received limited courses of antibiotics ranging from 2 to 4 weeks, without chronic suppression. All patients had their CIEDs removed prior to discharge, except case 3 where the patient eventually underwent LVAD exchange and CIED removal after failure of medical therapy. All patients except case 6 had their CIED reimplanted on the contralateral side of the chest. Case 6 no longer required an ICD. Those with CIED-IE had their CIED reimplanted after blood cultures were negative for a minimum of 7 days. Those with pocket infection had a device reimplanted after 48 hours or longer of having negative blood cultures.

The mean \pm SD time to follow-up for the entire cohort of 247 patients with LVADs in our study was $1.3 \pm 1.1$ years while the median follow-up duration was 1 year. All patients with CIED infection, except case 1, were alive at the last follow-up, with median duration of 15 months (range, 7 to 46 months).

\section{DISCUSSION}

The current investigation is the first to examine the clinical manifestations and management of CIED infections in LVAD recipients, a unique combination of devices that was seen in the majority of patients who underwent LVAD placement in our medical center and a scenario that will likely increase as an increasing number of LVADs are being implanted as destination therapy for end-stage heart failure. The CIED infection rate of two percent is similar to that seen in non-LVAD patients (1.53\% to $2.41 \%$ ), based on a recent report that utilized the Nationwide Inpatient Sample (NIS) discharge records [12]. Previous work form our institution has shown that CIED-IE is more frequent in patients with S. aureus BSI and underlying prosthetic heart valves [13]. Larger cohorts are needed to determine if this is true for patients with both CIED and LVAD as well. 
Most CIED infections in our series presented late (16-18 months after LVAD implantation), suggesting that the LVAD implantation procedure did not increase CIED infection risk. Clinical manifestations of CIED infections in LVAD recipients appear quite similar to those without LVADs. Patients with CIED pocket infections presented with local inflammatory signs at the generator site while those with CIED-IE presented with BSI and echocardiographic evidence of lead vegetations (Table 1). The tricuspid valve involvement in one case is consistent with prior observation that the tricuspid valve is the most frequently infected valve in cases of CIED-IE [9]. However, the reflective surface of an LVAD may impair visualization of a vegetation on TEE examination [14]. Therefore, it is conceivable that some cases of CIED-IE may have been missed in our series.

The microbiologic etiologies of CIED infections in our series are similar to those seen in patients without LVADs [8]. All three patients with CIED-IE had prior LVAD-related infections and two of these had prior BSI due to the same organism that caused subsequent CIED infection. Case 1 demonstrated the progression of an initial driveline infection leading to transient BSI that ultimately seeded the CIED lead and the LVAD endovascular surface, all from the same organism. These observations suggest that LVAD-related infections, in particular BSI, can lead to the hematogenous seeding of CIED leads, resulting in increased likelihood of developing CIED-IE. However, the development of CIED pocket infections does not appear to be associated with LVAD-related infections.

The management of CIED infections in patients with LVAD warrants special consideration as illustrated in this case series. CIED pocket infections can be managed by device removal, implantation on the contralateral side once blood cultures are reported negative, and a limited course of systemic antibiotic therapy (10 - 14 days), similar to those without LVADs. CIED-IE, in contrast, presents a unique challenge because the endovascular surface of the LVAD is presumably seeded. It is unclear if there is a window of opportunity in an LVAD patient with BSI to remove the CIED before the LVAD is seeded. As previously demonstrated, conservative management of CIED-IE, without lead removal, is ineffective and leads to relapse or persistent infections [15, 16]. However, in LVAD patients, CIED lead removal still leaves an infected device (i.e. LVAD) in place, hence requiring chronic suppressive antibiotic therapy. Ideally, concurrent removal of LVAD and CIED would likely provide the best chance for cure of infection. However, LVAD removal is associated with increased morbidity and mortality, requiring either placement of a new device in an infected operative field (which would still require chronic antibiotic suppression) or a heart transplant. Case 3 in our series demonstrated the poor outcome with relapsing infection after the infected CIED was left in place, eventually requiring complete CIED and LVAD replacement. However, this case was confounded by the difficult-to-treat small colony variants of MRSA that probably led to persistent device infection. With aggressive device removal in most patients in our series, outcomes were generally good with all but one patient alive at the last follow-up, with median of 15 months (range 7-46 months) from onset of CIED infection.

Whether LVAD recipients still require CIED after LVAD implantation, when many were placed for cardiac resynchronization therapy for advanced heart failure, should be considered before re-implanting a new device in cases with CIED infection. It is conceivable that CIED therapy may no longer be necessary in some patients, thus avoiding dual devices in these patients at high risk of infectious complications.

In conclusion, management of CIED infection in LVAD recipients warrants special consideration. While CIED pocket infection can be managed with device removal and a limited course of antibiotic therapy, the endovascular surface of LVAD should be presumed seeded in cases of CIED infection complicated by BSI or CIED-IE. These cases should be 
managed with chronic suppressive antibiotic therapy until the infected LVAD is removed and replaced with a new device or until the patient undergoes heart transplantation. Also, aggressive management of infection, with prompt CIED removal and antibiotic therapy, is crucial to prevent subsequent systemic infection. Failure to remove all infected or colonized hardware (LVAD and/or CIED) is associated universally with failure of treatment. Larger studies are needed to better define infection risk, optimal management strategies, and estimate survival in this unique group of patients with dual cardiac devices.

\section{Acknowledgments}

This work was supported by a Career Development Award to Dr. Sohail from the Department of Medicine, Mayo Foundation for Medical Education and Research and the Small Grants award from the Division of Infectious Diseases, Mayo Clinic College of Medicine, and NIH/NCRR CTSA Grant Number UL1 RR024150. The study database was created and maintained using REDCap (Grant Support UL1 TR000135). Its contents are solely the responsibility of the authors and do not necessarily represent the official views of the NIH.

\section{References}

1. Mond HG, Proclemer A. The 11th world survey of cardiac pacing and implantable cardioverterdefibrillators: calendar year 2009--a World Society of Arrhythmia's project. Pacing Clin Electrophysiol. 2011 Aug; 34(8):1013-1027. [PubMed: 21707667]

2. Thoratec. HeartMate-II-Fact-Sheet-B100-0611-FINAL. [Fact Sheet Article]. 2011. [updated Nov 3, 2011]; Available from: http://www.thoratec.com.

3. Rose EA, Gelijns AC, Moskowitz AJ, Heitjan DF, Stevenson LW, Dembitsky W, Long JW, Ascheim DD, Tierney AR, Levitan RG, Watson JT, Meier P, Ronan NS, Shapiro PA, Lazar RM, Miller LW, Gupta L, Frazier OH, Desvigne-Nickens P, Oz MC, Poirier VL. Long-term use of a left ventricular assist device for end-stage heart failure. N Engl J Med. 2001 Nov 15; 345(20):14351443. [PubMed: 11794191]

4. Duru F, Candinas R, Lachat M, Rahn M, Noll G, Luscher TF, Turina M. Electrical and mechanical support in advanced heart failure. Rationale and feasibility of a combined management strategy. Eur Heart J. 2002 Jul; 23(13):1005-1010. [PubMed: 12093052]

5. Le KY, Sohail MR, Friedman PA, Uslan DZ, Cha SS, Hayes DL, Wilson WR, Steckelberg JM, Baddour LM. Clinical predictors of cardiovascular implantable electronic device-related infective endocarditis. Pacing Clin Electrophysiol. 2011 Apr; 34(4):450-459. [PubMed: 21208230]

6. Hannan MM, Husain S, Mattner F, Danziger-Isakov L, Drew RJ, Corey GR, Schueler S, Holman WL, Lawler LP, Gordon SM, Mahon NG, Herre JM, Gould K, Montoya JG, Padera RF, Kormos RL, Conte JV, Mooney ML. Working formulation for the standardization of definitions of infections in patients using ventricular assist devices. J Heart Lung Transplant. 2011 Apr; 30(4): 375-384. [PubMed: 21419995]

7. Sohail MR, Uslan DZ, Khan AH, Friedman PA, Hayes DL, Wilson WR, Steckelberg JM, Jenkins SM, Baddour LM. Infective endocarditis complicating permanent pacemaker and implantable cardioverter-defibrillator infection. Mayo Clin Proc. 2008 Jan; 83(1):46-53. [PubMed: 18174000]

8. Sohail MR, Uslan DZ, Khan AH, Friedman PA, Hayes DL, Wilson WR, Steckelberg JM, Stoner S, Baddour LM. Management and outcome of permanent pacemaker and implantable cardioverterdefibrillator infections. J Am Coll Cardiol. 2007 May 8; 49(18):1851-1859. [PubMed: 17481444]

9. Baddour LM, Epstein AE, Erickson CC, Knight BP, Levison ME, Lockhart PB, Masoudi FA, Okum EJ, Wilson WR, Beerman LB, Bolger AF, Estes NA 3rd, Gewitz M, Newburger JW, Schron EB, Taubert KA. Update on cardiovascular implantable electronic device infections and their management: a scientific statement from the American Heart Association. Circulation. 2010 Jan 26; 121(3):458-477. [PubMed: 20048212]

10. Horan TC, Andrus M, Dudeck MA. CDC/NHSN surveillance definition of health careassociated infection and criteria for specific types of infections in the acute care setting. Am J Infect Control. 2008; 36(5):309-332. [PubMed: 18538699]

11. K/DOQI clinical practice guidelines for chronic kidney disease: evaluation, classification, and stratification. Am J Kidney Dis. 2002 Feb; 39(2 Suppl 1):S1-S266. [PubMed: 11904577] 
12. Greenspon AJ, Patel JD, Lau E, Ochoa JA, Frisch DR, Ho RT, Pavri BB, Kurtz SM. 16-year trends in the infection burden for pacemakers and implantable cardioverter-defibrillators in the United States 1993 to 2008. J Am Coll Cardiol. 2011 Aug 30; 58(10):1001-1006. [PubMed: 21867833]

13. Uslan DZ, Dowsley TF, Sohail MR, Hayes DL, Friedman PA, Wilson WR, Steckelberg JM, Baddour LM. Cardiovascular implantable electronic device infection in patients with Staphylococcus aureus bacteremia. Pacing Clin Electrophysiol. 2010 Apr; 33(4):407-413. [PubMed: 19793360]

14. Nurozler F, Argenziano M, Oz MC, Naka Y. Fungal left ventricular assist device endocarditis. Ann Thorac Surg. 2001; 71(2):614-618. [PubMed: 11235716]

15. Chua JD, Wilkoff BL, Lee I, Juratli N, Longworth DL, Gordon SM. Diagnosis and Management of Infections Involving Implantable Electrophysiologic Cardiac Devices. Ann Intern Med. 2000 Oct 17; 133(8):604-608. 2000. [PubMed: 11033588]

16. Klug D, Lacroix D, Savoye C, Goullard L, Grandmougin D, Hennequin JL, Kacet S, Lekieffre J. Systemic Infection Related to Endocarditis on Pacemaker Leads : Clinical Presentation and Management. Circulation. 1997 Apr 15; 95(8):2098-2107. 1997. [PubMed: 9133520] 


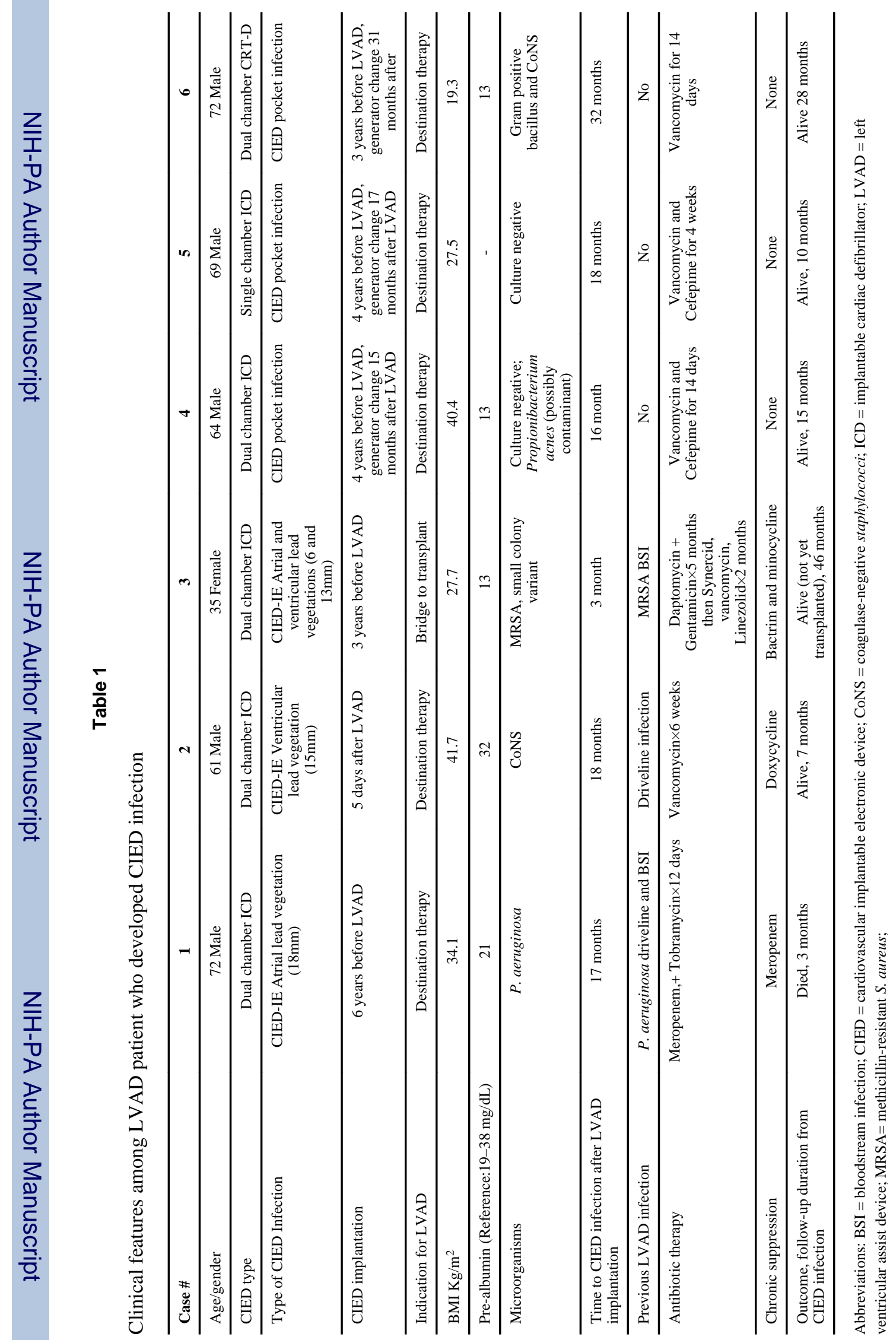

Pacing Clin Electrophysiol. Author manuscript; available in PMC 2015 February 01. 